Revue d'histoire des chemins de fer

\title{
Ouverture du colloque
}

Gérard Mayaud

\section{OpenEdition}

\section{Journals}

Édition électronique

URL : https://journals.openedition.org/rhcf/2018

DOI : 10.4000/rhcf.2018

Éditeur

Rails \& histoire

Édition imprimée

Date de publication : 2 mai 2002

Pagination : 7-8

ISBN : 00996-9403

ISSN : 0996-9403

\section{Référence électronique}

Gérard Mayaud, "Ouverture du colloque », Revue d'histoire des chemins de fer [En ligne], 24-25 | 2002,

mis en ligne le 02 février 2015, consulté le 22 avril 2022. URL : http://journals.openedition.org/rhcf/

2018 ; DOI : https://doi.org/10.4000/rhcf.2018 


\section{Gérard MAYAUD \\ Ouverture du colloque}

Mesdames, Messieurs,

Je vous souhaite la bienvenue au Centre universitaire de Châteauroux où j'ai le plaisir d'accueillir le $9^{\mathrm{e}}$ colloque de l'Association pour 1'histoire des chemins de fer en France : «Le Chemin de fer à la conquête des campagnes : l'aménagement du territoire par les réseaux dits « secondaires » en France, histoire et patrimoine, 1865-2001 ».

Il a la particularité de se réunir le jour même de la $14^{\mathrm{e}}$ rentrée universitaire à Châteauroux. Aujourd'hui commencent en effet les cours du Centre d'études supérieures de l'Université d'Orléans dans trois filières : Droit, Administration économique et sociale et Économie de gestion. Le $1^{\text {er }}$ octobre 2001 le Centre accueillera les étudiants en histoire, en géographie et en langues étrangères appliquées. Un nouveau cursus commencera le 22 octobre prochain, dont l'objet n'est pas étranger à vos réflexions, puisqu'il s'agit de former des spécialistes de l'aménagement et du développement des territoires ruraux.

Ce colloque s'inscrit dans la tradition de ceux qu'organise en ces lieux le Centre de recherche, d'étude et de documentation de l'Indre ${ }^{1}$. Le C.R.E.D.I. consacre en effet sa prochaine rencontre (7-8 décembre 2001) à l'avenir des espaces ruraux. C'est un problème essentiel, auquel votre colloque fournit des références en nous rappelant l'histoire des réseaux secondaires. Pour ma part, je ne peux que regretter que l'on ait vu disparaitre progressivement la plupart des lignes, même celles qui reliaient deux chefs-lieux comme Tours et Châteauroux.

Alors que l'aménagement du territoire et la sécurité des déplacements sont des priorités pour chacun on ne peut que constater l'incohérence des politiques adoptées avec ces objectifs : l'irrigation des territoires par les transports publics est, il faut hélas le reconnaitre, de plus en plus déficiente dès que l'on s'éloigne du réseau qui relie la capitale aux grandes villes régionales et départementales. Celui-ci n'est pas non plus sans poser un problème d'actualité, à savoir la mise en place d'un service de trains pendulaires sur l'itinéraire Paris-Orléans LimogesToulouse (POLT) dont l'Indre attend beaucoup. 
Quoi qu'il en soit, je souhaite profiter de cet instant pour renouveler publiquement les remerciements que le Conseil général et moi-même, au nom du Centre universitaire, adressons au professeur Wolkowitsch, responsable scientifique de ce colloque.

Vous avez, Monsieur le Professeur, choisi Châteauroux pour organiser ce colloque sur un sujet dont vous êtes le spécialiste et qui vous tient particulièrement à cœur, après avoir doté le Centre universitaire de votre bibliothèque personnelle. Sachez que les enseignants et les étudiants de géographie et d'aménagement vous en sont très reconnaissants et qu'ils feront le meilleur usage de ce don qui renforce considérablement notre fonds dans ce domaine.

J'ouvre les débats en vous souhaitant à tous une excellente et fructueuse journée.

\section{Note}

1- Par exemple : Le Préfet d'hier à aujourd'hui, actes du colloque de Châteauroux des 18 et 25 mars 2000, Châteauroux, Ed. du C.R.E.D.I. (15, rue de la République, 36000 Châteauroux), 2001, 212 p. ; voir aussi : Les Séminaires, collection des actes des rencontres du C.R.E.D.I. : « Sociabilité et vie politique en Berry (Indre et Cher), XVIII ${ }^{e}$ - XXe siècle », 1999; " Annuaire de la recherche scientifique dans l'Indre », 2001. Le C.R.E.D.I. publie également une Bibliographie berrichonne annuelle. 